IOS Press

\title{
Review
}

\section{COVID-19 pandemic: Insights into genetic susceptibility to SARS-CoV-2 and host genes implications on virus spread, disease severity and outcomes}

\author{
Saba Dastar ${ }^{\mathrm{a}}$, Jalal Gharesouran ${ }^{\mathrm{b}}$, Deniz Mortazavi ${ }^{\mathrm{c}}$, Hassan Hosseinzadeh ${ }^{\mathrm{b}}$, Seyed Jalal Kian ${ }^{\mathrm{d}}$, \\ Mohammad Taheri ${ }^{\mathrm{e}}$, Soudeh Ghafouri-Fard ${ }^{\mathrm{f}}$, Elena Jamali ${ }^{\mathrm{g}, *}$ and Maryam Rezazadeh ${ }^{\mathrm{b}, *}$ \\ ${ }^{a}$ Division of Cancer Genetics, Department of Basic Oncology, Oncology Institute, Istanbul University, Fatih, \\ Istanbul, Turkey \\ ${ }^{\mathrm{b}}$ Department of Medical Genetics, Faculty of Medicine, Tabriz University of Medical Sciences, Tabriz, Iran \\ ${ }^{\mathrm{c}}$ Department of Genetics and Molecular Biology, School of Medicine, Isfahan University of Medical Sciences, \\ Isfahan, Iran \\ ${ }^{\mathrm{d}}$ Department of Virology, School of Medicine, Iran University of Medical Sciences, Tehran, Iran \\ e Skull Base Research Center, Loghman Hakim Hospital, Shahid Beheshti University of Medical Sciences, Tehran, \\ Iran \\ ${ }_{\mathrm{f}}^{\mathrm{f}}$ Department of Medical Genetics, School of Medicine, Shahid Beheshti University of Medical Sciences, Tehran, Iran \\ ${ }^{\mathrm{g}}$ Department of Pathology, Loghman Hakim Hospital, Shahid Beheshti University of Medical Sciences, Tehran, Iran
}

\begin{abstract}
The outbreak of the newly emerging severe acute respiratory syndrome coronavirus 2 (SARS-CoV-2) all over the world has caused global public health emergencies, international concern and economic crises. The systemic SARS-CoV-2 disease (COVID-19) can lead to death through causing unrestrained cytokines-storm and subsequent pulmonary shutdown among the elderly and patients with pre-existing comorbidities. Additionally, in comparison with poor nations without primary health care services, in developed countries with advanced healthcare system we can witness higher number of infections per one million people. In this review, we summarize the latest studies on genes associated with SARS-CoV-2 pathogenesis and propose possible mechanisms of the virus replication cycle and its triggered signaling pathways to encourage researchers to investigate genetic and immune profiles of the disease and try strategies for its treatment. Our review shows that immune response in people with different genetic background might vary as African and then Asian populations have lowest number of affected cases compared with European and American nations. Considering SARS-CoV-2 pathogenesis, we put forward some potentially important genetic gateways to COVID-19 infection including genes involved in the entry and replication of SARS-CoV-2 and the regulation of host immune response which might represent explanation for its spread, severity, and morality. Finally, we suggest that genetic alterations within these gateways could be critical factors in influencing geographical discrepancies of the virus, so it is essential to fully study them and design appropriated and reliable therapeutic agents against COVID-19.
\end{abstract}

Keywords: COVID-19, SARS-CoV-2, coronavirus, host gene, susceptibility

*Corresponding authors: Elena Jamali, Department of Pathology, Loghman Hakim Hospital, Shahid Beheshti University of Medical Sciences, Tehran, Iran. E-mail: Elena.jamali@yahoo.com. Maryam
Rezazadeh, Department of Medical Genetics, Faculty of Medicine, Tabriz University of Medical Sciences, Tabriz, Iran. E-mail: Rezazadehm@tbzmed.ac.ir. 


\section{Introduction}

Coronavirus disease 2019 (COVID-19) is the most recent pandemic threat to global public health, caused by severe acute respiratory syndrome coronavirus 2 (SARS-CoV-2) [1,2]. Incubation period of the disease is 2-14 days and it associates with symptoms such as respiratory distress, dyspnea, dry cough, conjunctivitis, fever, diarrhea, acute kidney failure, and loss of taste and smell. Bats (Rhinolophus) are known as primary host and Malayan pangolin has been considered as possible intermediate host [3].

The virus belongs to the Coronaviridae family that are relatively large in size $(50-200 \mathrm{~nm}$ in diameter) [4]. These viruses have a membrane which covers a long single-stranded positive-sense ribonucleic acid (RNA) genome $(\sim 30 \mathrm{~kb})$. The virion membrane contains both host and viral proteins and glycoproteins, including spike glycoproteins that protrude from the surface of the virus and give it the crown-like appearance under electron microscope. These viruses can spread from animal hosts such as bats, civet and cats to human and cause life-threatening respiratory infections $[2,5]$.

\subsection{Coronavirus classification/taxonomy}

Coronaviruses are classified under the family Coronaviridae, that is the largest of three RNA virus families within the order Nidovirales [3]. The family is divided into two subfamilies, Orthocoronavirinae and subfamily Torovirinae [6]. The Torovirinae subfamily contains viruses that cause mainly enteric infections in cattle, horses, pigs, and cats, but they are not yet known to cause human infection. According to genotypic and serological characterization, members of the subfamily Orthocoronavirinae are subdivided into four genera including Alphacoronavirus, Betacoronavirus, Gammacoronavirus, and Deltacoronavirus [3]. Alpha and betacoronaviruses are harbored in mammals, particularly bat, causing gastroenteritis in animals and respiratory illnesses in humans. Gammacoronaviruses mostly circulate in avian species and a few mammals, whereas Deltacoronavirus infect birds and mammalian specious [3,7]. Among all coronaviruses, seven of them cause human diseases. Four of these viruses cause common cold symptoms especially mild respiratory infection in immunocompromised individuals; these coronaviruses are $\mathrm{HCoV} 229 \mathrm{E}, \mathrm{HCoV}$ OC43, HCoV NL63, and HCoV HKU1 [8]. The remaining three strains which cause severe respiratory dis- tress/pneumonia and even death are SARS-CoV-1, Middle Eastern respiratory syndrome (MERS)-CoV, and SARS-CoV-2 [8].

\subsection{Structure of SARS-CoV-2}

The RNA genome of the virus is around $30 \mathrm{~kb}$ in size, capped at the 5' end, 3'-polyadenylated and contains two flanking untranslated regions (UTRs). It has multiple open reading frames (ORFs) which encode several genes. The order of the genes in the genome is a noncoding 5'-UTR, replicase genes (two overlapping ORFs (ORF1a and 1b)), structural proteins (spike (S), envelope (E), membrane (M), and nucleocapsid (N)) and accessory proteins-noncoding 3'-UTR [8,9]. The ORF1a and $1 \mathrm{~b}$ which are considered as the largest ORF, encode for 15 nonstructural proteins (nsp) (nsps 1 to 10 and nsps 12 to 16) [9]. These ORFs are translated into two polypeptides, ppla and pplab that are identical at the $\mathrm{N}$ terminal while one of them has a $\mathrm{C}$-terminus extension because of a frame-shifting event. Two cysteine proteases including nsp3 and nsp5 also known as a papain-like protease (PL2pro) and 3C-like protease (3CLpro), respectively, are produced as well. The nsp3 is responsible for cleaving between nsp1 $|2, n s p 2| 3$, and nsp3|4 sites, and the nsp5 breaks the LQ $\mid$ SAG sites to produce nsp4 through nsp16. Among all ORF1a and $1 \mathrm{~b}$ encoded nsps, nsp7, nsp8 in complex with nsp12 (RNA-dependent RNA polymerase (RdRP)), nsp13 (helicase), and nsp14 (exonuclease) are the most important viral proteins as they involve in transcription and viral RNA replication $[10,11]$. The nsp13 not only has helicase activity but also has NTPase, dNTPase, and 5' triphosphatase activities [12].

The 3'-end of the virus genome encodes four structural proteins that are critical for binding to host cell receptor, assembly of the virion, morphogenesis, and release of mature virus particles from the host cell. The SARS-CoV-2 surface (S) protein is a transmembrane heavily glycosylated protein that has three major parts; $\mathrm{S} 1$ region contains a $\mathrm{NH} 2$-terminal domain (NTD) and a C-terminal domain (CTD) also known as the receptorbinding domain (RBD), S2 subunit or stalk fusion domain which is a transmembrane domain (TM), and a short cytoplasmic tail region (CP). The ectodomain of $S$ protein forms homotrimeric spikes on the viral membrane surface and mediates viral entry into host cells. This protein is considered as a multifunctional molecular machine that has important roles in the early steps of viral infection through interaction with host susceptibility factors such as receptors and proteases [12-15]. The 
transmembrane $\mathrm{M}$ glycoprotein is the most abundant protein in the viral particle. On the contrary, while the E protein is found in small quantities in the viral membrane, it is abundantly expressed inside the host cell, localized mainly in endoplasmic reticulum (ER) and Golgi complex of the infected cells $[16,17]$. The difference in the number of these proteins suggests that $M$ protein along with E protein is critical for arranging the assembly of the virus and forming its envelope [18]. In addition to other functions, the E protein as the smallest of all structural proteins is involved in release of viral particles from host cells $[19,20]$. During viral assembly, the $\mathrm{N}$ protein is responsible for packaging of the viral genome into a helical ribonucleocapsid (RNP) through binding to the viral RNA [21,22]. Furthermore, SARSCoV-2 also has multiple subgenomic RNAs, including $3 \mathrm{a}, 3 \mathrm{~b}, 6,7 \mathrm{a}, 7 \mathrm{~b}, 8 \mathrm{~b}, 9 \mathrm{~b}$, and orf 14 that are distributed along the structural genes and encode eight accessory proteins $[9,23]$.

\subsection{SARS-CoV-2 entry}

According to molecular modeling studies, RBD domain of $\mathrm{S}$ protein has a strong binding affinity to human angiotensin-converting enzyme 2 (ACE2) [24,25]. ACE2 is generally localized in the surface of cells of the lung, kidney, heart, and small intestine, among others. Up to the present time, ACE2 has been suggested as the most possible receptor candidate for the virus. However, there is a need for further investigation of the receptor usage, ligand activation, viral pathogenesis and also the tropism of the virus. In a model study, it has been shown that CTD residues A475, N487, E484, and Y453 interact with S19, Q24, K31, and $\mathrm{H} 34$ of the $\alpha 1$ helix of ACE2, respectively and form strong polar contacts. Additionally, residues Q498, T500, and N501 on the bulged loop form a solid network of H-bonds with Y41, Q42, K353, and R357 from ACE2 [8,26,27].

After binding of ACE2 to RBD, the trimeric S protein is cleaved by host cell proteases that make the $\mathrm{S} 2$ subunit of the virus to undergo some conformational changes, which subsequently leads to the fusion of the viral envelope with target cell membrane [28-30]. The S2 subunit of the virus has two heptad repeat regions 1 and 2 (HR1 and HR2) which are essential for the virus membrane fusion with the host cell. In different coronaviruses, the cleavage of $S$ protein occurs at between the $\mathrm{S} 1$ and $\mathrm{S} 2$ domains ( $\mathrm{S} 1 / \mathrm{S} 2$ site) and also within the S2 domain proximal to the fusion peptide (S2 site) $[31,32]$. It seems that both sites are needed to be cleaved for virus entry. Based on amino acid sequence in different viruses, different proteases are needed to cleave the S1/S2 site. The S1/S2 site of SARS-CoV-2 (AYT $\downarrow \mathrm{M}$ ) is similar to the one in SARS-CoV that has been shown to be cleaved by cathepsin L $[33,34]$. Also, a furin-like protease cleavage S2' site (KR $\downarrow$ SF) exists in SARS-CoV-2 that is identical to that in SARS$\mathrm{CoV}$ and it is probably cleaved by cathepsin L or transmembrane serine protease 2 (TMPRSS2) in different cellular locations during viral entry. Previous studies have revealed that cathepsin L and TMPRSS2 inhibition suppressed virus entry and infection of permissive cells $[34,35]$. SARS-CoV-2 also has an additional cleavage site in N-terminus of the S1/S2 site (AYT $\downarrow$ M) known as furin-like protease cleavage site (RRAR $\downarrow$ SV), suggesting that it may be cleaved by furin during viral egress $[3,33]$.

\subsection{Viral replication}

After endosomal pH-dependent fusion of viral envelope with host cell membrane, the positive-strand genomic RNA is released into the cytoplasm and the translation of the replicase gene is started [29,36,37]. ORF1a cap-dependent translation generates replicase ppla polyprotein. Meanwhile, the presence of a slippery sequence (5'-UUU AAAC-3') and an RNA pseudoknot towards the end of ORF1a makes ribosomal frameshifting possible which leads to the production of a long pplab polyprotein. Both translated polyproteins undergo autoproteolytic cleavages which produce nsps, 1 to 11 and 1 to 16 for ppla and pplab, respectively. At the next step, the replicase-transcriptase complex (RTC) mediates the production of full length negativesense RNA copies of the viral genome which are used as templates for the production of full length positivesense RNA genomes. Subsequently, multiple copies of subgenomic RNA (sgRNA) species are produced by a mechanism known as discontinuous transcription. $\mathrm{sgR}$ NAs encode for different viral proteins and only their nearest ORF to 5' end is translated. After genome replication and sgRNAs translation, the structural proteins are entered into endoplasmic reticulum (ER) and migrate inside the secretory pathway within the ER-Golgi intermediate compartment (ERGIC). Concurrently, nucleocapsids assembled in the cytoplasm bud into ERGIC membranes that encompasses structural proteins. Through this process the life cycle of SARS-CoV-2 is completed and mature virions are released out of the cell via exocytosis [38-40].

Successful virus assembly is dependent on precise 


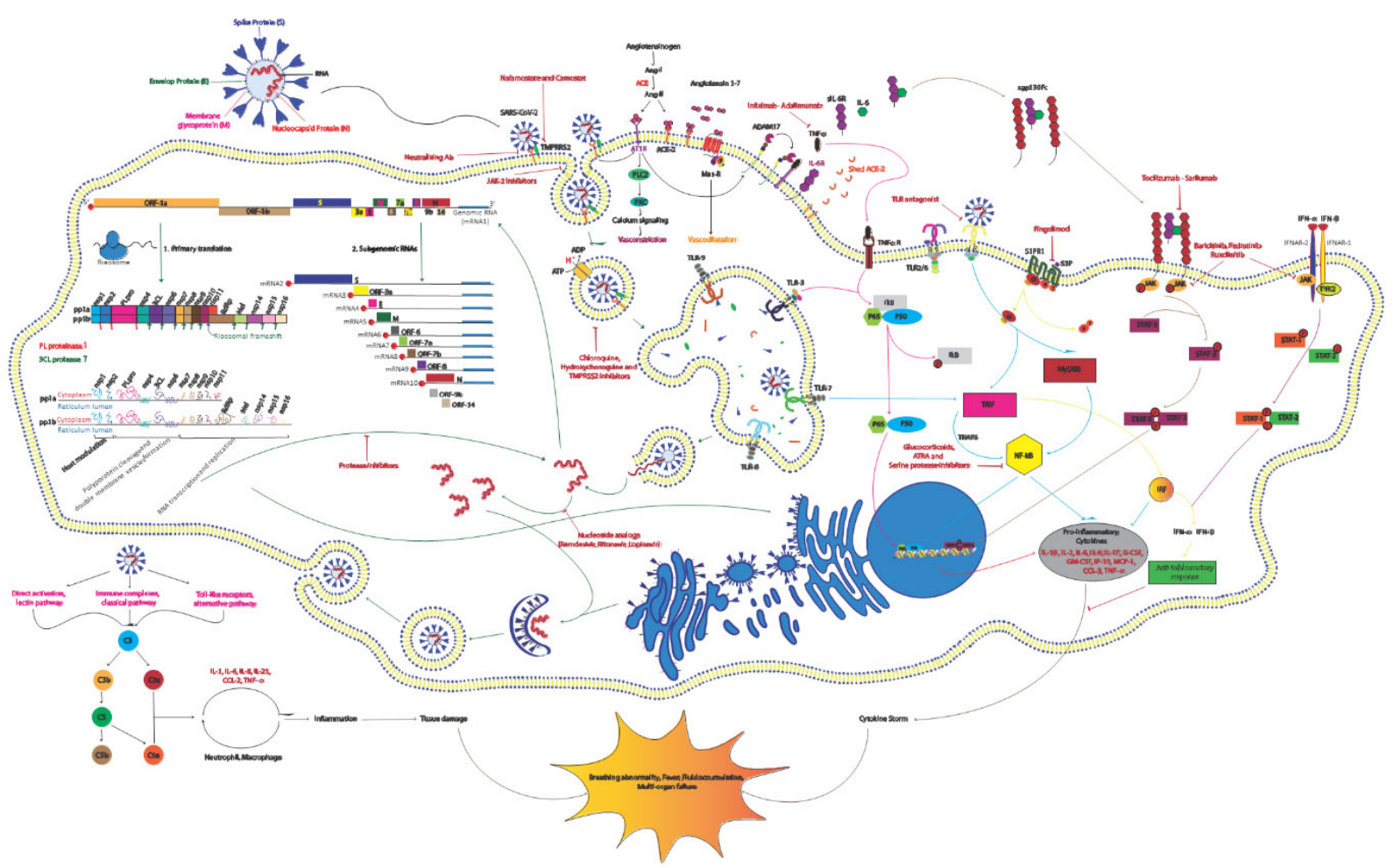

Fig. 1. Schematic representation of the SARS-CoV-2 replication cycle, its driven signaling pathways and potential antiviral drugs. The life cycle of the virus in the target cells is shown with green arrows and signaling pathways associated with the host immune response are displayed with pink, blue, gray and brown arrows. As can be seen, the virus first enters host cells through binding to its known receptor angiotensin-converting enzyme 2 (ACE2) on the host cell membrane. Then the transmembrane serine protease 2 (TMPRSS2) processes viral spike protein (S) which leads JAK-2-mediated endocytosis and subsequently acidification of the endosome result in viral-host cell membrane fusion and release of viral single-stranded RNA (ssRNA) into the cytosol. Then host machinery translates the viral genome to produce the replicase and structural proteins. Initially open reading frame $1 \mathrm{a}(\mathrm{ORF} 1 \mathrm{a})$ and ORF1b are translated by ribosomal frameshifting into polyproteins pp1a and pp1ab, respectively, that are cleaved by the proteases papain-like protease (PLpro) and 3C-like protease (3CLpro) into 15 mature nonstructural proteins (nsps). Structural proteins are expressed as subgenomic RNAs which are created by discontinous transcription. Both genomic and subgenomic RNAs are translated to yield only the protein encoded by the 5'-most ORF. After viral replication and ampli?cation by the RNA-dependent RNA polymerase (RdRp), the newly produced RNAs are encapsidated in the nucleocapsid $(\mathrm{N})$ proteins in the cytoplasm and then transported to the ER-Golgi intermediate compartment (ERGIC) for further assembly. All structural proteins are inserted into the membrane of the rough ER (RER), from where they are shuttled to the ERGIC to interact with the viral RNA-N complex and assemble into viral particles. Finally, the budded vesicles containing the newly synthesized viral particle are transported to the cell surface for release (Green Arrows). Angiotensin II (Ang II) as a ligand of both AT1R and ACE2 is produced through ACE-mediated Ang I cleavage. By binding to AT1R pro-inflammatory response are activated and through attachment to ACE2, Ang II is converted to angiotensin 1-7 (Ang 1-7) that induce the Mas receptor associated anti-inflammatory response. Viral binding promotes shedding of proinflammatory cytokines and ACE2 receptors by some metalloproteases like ADAM17. The cleavage of ectodomains of these receptors makes them soluble which leads to loss of the protective function of surface ACE2 and may increase viral pathogenesis (Black Arrows). The virus activates nuclear factor $\kappa \mathrm{B}$ (NF-kB) signaling pathway and stimulates tumor necrosis factor $\alpha$ (TNF $\alpha$ ) and interleukin 6 (IL-6) release through degradation of $\mathrm{i} \kappa \mathrm{B}$ (Pink Arrows). Additionally, the virus stimulates massive release of cytokines which is known as cytokine storm through JAK/STAT, NF- $\kappa$ B and also toll-like receptors (TLRs) mediated pathways (Blue and Brown Arrows). Complement activation may contribute to the maladaptive inflammatory response. C3a and C5a are known pro-inflammatory molecules that promote immune cell recruitment to the site of infection (Black Arrows). Both cytokine storm and complement activation can lead to tissue damage and probable COVID-19 symptoms. However, the induction of the S1P/S1PR1 axis prevents cytokine storm associated with virus-induced pathology (Yellow Arrows). Additionally, Type I IFNs (IFN- $\alpha / \beta$ ) elicit the JAK/STAT signaling pathway through binding to IFNAR receptors (IFNAR1 and IFNAR-2). In this process AK1 and TYK2 phosphorylate STAT1 and STAT2, which in complex with IRF9 lead to the expression of interferon-stimulated genes (ISGs) and anti-viral status (Purple Arrows).

protein-protein interactions which are orchestrated by $\mathrm{M}$ and $\mathrm{E}$ proteins. $\mathrm{N}$ proteins seem essential for virus like particle (VLP) production, suggesting that nucleocapsid assembly and its fusion into ERGIC membranes promotes the viral particle formation. At this step, $\mathrm{S}$ proteins are incorporated into the particle and subsequent steps of the virions assembly is enhanced by $\mathbf{M}$ protein-nucleocapsid interaction [40,41] (Fig. 1). 


\section{The role of host genetic factors in COVID-19 infection}

Disease severity among affected patients without certain underlying medical conditions proposes the involvement of genetic susceptibility factors which makes some people more prone to severe symptoms. Therefore, the role of both individual and population genetics can be pivotal in shaping the COVID-19 dynamics. As a result, host genes associated with the entry and replication of the virus as well as mounting host immune responses should be assessed for reaching genetic predisposition models to COVID-19 disease [42].

\subsection{Population genomics role in COVID-19 pandemics}

The highest number of infections almost one year after the pandemic in January 2021 have been reported in Andorra $(107,656)$, followed by Gibraltar $(86,882)$, Montenegro (82,203), Luxembourg $(77,525)$ and other European countries. In this ranking system both number of affected people in a country and the number of cases per one million people is considered. So, the ranking system rules out population densities in some countries across continents. Although mentioned nations witnessed higher number of COVID-19 infection, most African and Asian countries even with wrecked health care system showed comparatively a smaller number of cases. With this regard, it seems that there is a geographical variation in infection to the virus, but the reasons behind it have been remained obscure. One of the proposing reasons for this pattern of infection can be differences in population genomics which provide susceptibility and protection against SARS-CoV-2 infection. Hence, understanding the host genetic background and differences between diverse populations are essential to investigate the risk and protective factors involved in developing the pandemic. Up to now, a new international COVID-19 host genetics initiative has been launched to give an opportunity to researchers all around the world for characterizing genetic determinants associated with COVID-19 susceptibility, severity, and outcomes.

\subsection{Genes involved in the entry and replication of SARS-CoV-2}

\subsubsection{ACE2 gene}

The ACE2 is located on chromosome Xp22.2 and expressed in main target organs of the virus and also other organs with less involvement or even unknown role in the viral pathophysiology. Both lung alveolar and small intestinal epithelial cells highly express ACE2 and take part in COVID-19 transmission. According to immune-localization studies, the protein is present in smooth muscle cells, vascular endothelial cells, basal epidermal layer of the skin, as well as oral and nasal mucosa [43]. In the kidney, ACE2 is expressed weakly or moderately in podocytes and parietal epithelial cells whereas in the brush border of proximal tubular cells it exists in extremely high quantity [44]. The encoded enzyme mediates the conversion of angiotensin II to angiotensin [1-7] that is a vasodilator agent and has a pivotal function in the cardiovascular system. Based on comparative genetic analysis, the distribution pattern and influence of some single nucleotide polymorphisms (SNPs) in coding regions of the gene showed varying allele frequencies in various populations [45]. Distribution of pathogenic mutations in ACE2 differs among 9 populations, particularly around $40 \%$ and $55 \%$ of variants occur in African/African-American and Non-Finnish Europeans. Other populations including Latino/Admixed American, Finnish, East Asian, and South Asian carry $2-10 \%$ of such variants in ACE2 coding regions. On the contrary, prevalence of deleterious variants among Amish and Ashkenazi Jewish populations is zero [46]. These findings suggest that certain ACE2 alterations may provide potential resistance to COVID-19. Some variants which have the ability to inhibit ACE2-spike protein interaction may prevent virus entry into host cells. This is reported about alteration of residues p.Met383, p.Pro389 and p.Asp427 or in residues $\operatorname{Arg} 708 / 710 / 716$ that are located in the dimeric interface of the protein and are essential for TMPRSS2 cleavage $[47,48]$. On the other hand, ACE2 pathogenic mutations can disrupt the renin-angiotensin balance and vascular functions which can subsequently cause hypertension and diabetes mellitus, the most common comorbid conditions of the virus [45]. Moreover, previous studies showed that ACE2 has pivotal role in inflammatory processes and its genetic deficiency results in overexpression of cytokines and induction of vascular inflammation in mouse model with knocked-out ApoE [49,50]. Furthermore, the association of ACE2 expression with several immune signatures such as B, $\mathrm{T}$ and NK cells' markers and even interferon response has been reported in different human tissues [51]. Consequently, the role of deleterious ACE2 variants in susceptibility to virus infection is largely conflicting with considering the receptor involvement in virus entry into host cell and also post-infection downstream processes like inflammatory responses. 
One of the strongest risk factors for severe COVID19 is age advancing [52]. Although higher complication and fatality rates among elderly can be justified by gradually decreasing innate and adaptive immune responses, expression of ACE2 in the lungs with age increasing can also provide good explanation to the higher disease severity and mortality among older patients with COVID-19 [53,54]. Current research showed that men are more prone to COVID-19 infection than women [55]. One of the possible mechanisms for gender related susceptibility to COVID-19 is ACE2 localization on chromosome $\mathrm{X}$, in which monoallelic versus biallelic presence of the gene put men at higher risk of infection and severity $[46,56,57]$.

\subsubsection{TMPRSS2 gene}

The TMPRSS2 gene, also known as epitheliasin is located on chromosome 21q22.3 and encodes androgenresponsive serine protease which primes spike protein of SARS-CoV-2 and facilitates viral entry and activation $[58,59]$. This protein is a 492 amino acid singlepass type II membrane protein and contains five domains including cytoplasmic domain, a transmembrane domain (aa 84-106), an LDL receptor class A (LDLRA, aa 113-148) domain (forms a binding site for calcium), a Scavenger receptor cysteine-rich domain (SRDR, aa 149-242) and a Serine protease domain (aa 255492) [60]. Although the normal physiological function of the protein has been remained obscure, some studies have revealed that TMPRSS2 expression reduces the epithelial sodium channel and protein levels [60]. This enzyme is expressed in many tissues including lung, colon, kidney, cardiac endothelium, and small intestine; however, it is well known for its predominant expression in the prostate epithelium $[61,62]$. These findings suggest that clinical manifestations of the virus such as acute myocardial injury, gastrointestinal complications and thrombosis as a result of endothelial dysfunction are associated with expression of TMPRSS2 in the mentioned tissues $[63,64]$. Considering alteration frequencies of TMPRSS2 among different population, $35 \%$ and $59 \%$ of pathogenic variants are carried by African/African-American and Non-Finnish European populations, respectively. Meanwhile, only four deleterious variants are carried by each of the South Asian, East Asian and Finish populations [46]. A key residue for catalytic substrate binding of this enzyme is Asp435, which its substitution with Tyr has been seen with low frequency among Non-Finnish European population. These polymorphisms with diverse frequency provide potential explanation for differential genetic susceptibility to the virus.
Overexpression of this gene has been reported in association with some factors. Schuler et al. showed that in ciliated cells and type I alveolar epithelial cells (AT1) of human and mice there is an age-related expression pattern for TMPRSS2. They illustrated that expression level of the gene is increasing with age and suggested that developmental regulation of TMPRSS2 might be a good explanation for comparative protection of infants and children from the virus $[65,66]$. Considering chromosomal location of the TMPRSS2 gene, it seems that Down syndrome patients might be at higher risk due to overexpression of the gene. Its oncogenic role in some cancers might explain poor outcomes in COVID19 as well, particularly in individuals with prostate cancer $[45,49]$.

\subsubsection{Furin}

Second gene that adds an additional level of complexity in SARS-CoV-2 entry is furin. This gene is located on chromosome $15 \mathrm{q} 26.1$ and encodes a type 1 membrane-bound protease which is expressed in many organs including brain, lung, liver, pancreas, gastrointestinal tract, and reproductive tissues [67]. Cleavage of a polybasic site at the $\mathrm{S} 1-\mathrm{S} 2$ boundary of spike protein by the furin protease is thought to facilitate the entrance of the virus into its target cells $[14,67]$. Hoffmann et al. in a study on human lung cells described that the cellular protease furin is essential for S-protein-mediated cell-cell fusion and entry into host cells and they suggested that furin can be a potential target for therapeutic intervention [68]. Unlike TMPRSS2, furin is required for normal development, so its inhibition for prolonged time periods can have undesirable toxic effects $[68,69]$. In contrast, in other studies several variants both in the furin cleavage site and furin gene which may affect COVID-19 entrance have been studied and their results showed that the furin cleavage site might not be required for the virus to enter human cells in vivo $[67,70]$. With this regard, more studies are needed to clarify the exact role of furin in SARS-CoV-2 infection and also its polymorphisms effect on susceptibility to the virus among different populations.

\subsection{Immunogenetics in COVID-19}

\subsubsection{HLA}

The human leukocyte antigen (HLA) molecules are essential immune regulatory components with specific role in response to viral infections as receptors of viral peptides. These molecules are encoded by Major Histocompatibility Complex (MHC) genes and show ex- 
treme diversity and variations. Considering the fact that genetic differences at these genes result in various immune response against pathogens, HLA allele distribution among populations can provide a pattern for differential susceptibility to SARS-CoV-2 infection $[71,72]$.

A study on patients with severe respiratory distress, revealed that alongside with reduction of CD4, CD19 lymphocytes and NK cells, expression of HLA-DR was decreased to great extent, suggesting the involvement of HLA in COVID-19 progression [73]. A comprehensive in silico analysis for assessing binding affinity of all peptides of SARS-CoV-2 and MHC class I molecules across 145 HLA-A, -B, and -C genotypes revealed that individuals with HLA-B*46:01 alleles may not be vulnerable to the virus infection as this allele had the fewest affinity to the virus peptides. In contrast, HLA-B*15:03 had the greatest predicted binding peptides for SARSCoV-2 suggesting probable role of the allele in increasing susceptibility to covid-19 [74]. Analysis of SARS$\mathrm{CoV}$ and SARS-CoV-2 proteomes for prediction of the MHC class I epitope revealed that HLA-A*02:03 and $A * 31: 01$ are as effective antigen presenters for COVID19 indicating that these alleles might provide protection for their carrier individuals, whereas HLA-A*03:02 seems to be a risk factor [75]. However, in other studies multiple HLA alleles such as HLA-A*02:01, HLA-A*02:02, HLA-B*15:03, HLA-B*40:01, HLAC*12:03, HLA-DRA*01:01, HLA-DRB1*07:01, and HLA-DRB 1*04:01 have been reported in association with some epitopes of SARS-CoV-2 [74,76,77]. Considering all this, the pivotal role of $\mathrm{MHC}$ in developing anti-viral epitope-based peptide vaccine is substantial.

Population-specific HLA alleles is another concept for describing susceptibility of different populations to COVID-19. For instance, protective HLA-A*02 alleles were shown to have high frequencies among Indian African populations [78,79]. On the contrary, the susceptibility allele HLA-B*46:01 is highly distributed in South East Asia and rarely in Europe, while in Indian and African populations it is completely absent [80]. Another protective allele is HLA-B*15:03 which showed high frequency among African people, but it was absent in East Asian gene pool. Other allele that increases vulnerability to the virus is HLA$C^{*} 12: 03$. This HLA presumably is the most frequent allele among the European people [45]. These findings can be a good explanation for less risk and severity of the virus in Indian and African populations than European and other world populations and implicate that in people from different backgrounds, susceptibility according to HLA loci can vary significantly.

\subsubsection{SARS-CoV-2-driven signaling pathways}

Cytokine storm is an exacerbated systemic inflammatory response to COVID-19 which results in death due to multiple organ failure particularly lung, heart, kidney and liver $[81,82]$. This response occurs mainly because immune effector cells release a huge amount of pro-inflammatory cytokines and chemokines such as IL1- $\beta$, IL1RA, IL2, IL-6, IL7, IL8, IL9, IL10, IP10, IFN $\gamma$, GCSF, GMCSF, basic FGF2, VEGFA, MCP1, $\operatorname{MIP} 1 \alpha, \operatorname{MIP} 1 \beta, \mathrm{TNF} \alpha, \mathrm{PDGFB}$ and Vitamin D associated genes into blood [83-85] (Fig. 1). Immunologic cascade is associated with cytokine expression especially in $\mathrm{T}$ cells as prolific producers of cytokines. Both genetic and epigenetic processes are involved in development, differentiation, and activation of $\mathrm{T}$ cells and subsequent cytokines production. The levels of cytokines are in direct relation with various upstream regulators such as Toll-like receptors (TLR) and interaction with other components of innate immunity like complement components and also polymorphisms of cytokine genes which are distributed within populations with diverse patterns [86-89]. Some polymorphisms in cytokine gene confer vulnerability or protection to infections, so it seems that these polymorphisms can be risk factors for COVID-19 and influence the severity of the disease $[90,91]$.

Previous investigations revealed that polymorphisms of TLR genes or components of TLR pathway affect SARS-CoV infection. In animal models with deficiency of TLR3, TLR3/TLR4 adaptor TRIF and Toll Like Receptor Adaptor Molecule 2 (Ticam2), susceptibility to SARS-CoV infection has increased [92,93]. Variations in TLR genes result in different immunological redundancy of TLRs and distinct expression pattern in each population [94]. So, proposing TLR genes as a crucial determinant of differential vulnerability to COVID19 could be logical. However, this concept should be evaluated empirically.

As components of innate immunity, complement hyper-activation has been reported in COVID-19 patients [95]. In null C3 mice with SARS-CoV infection, levels of cytokines and chemokines in both the sera and lungs were low and respiratory dysfunction was not severe [96]. These reports implicate pivotal role of complement components in the induction of the cytokine storm and susceptibility to SARS-CoV-2 infection and disease severity. Although there is no evidence regarding the impact of complement gene polymorphisms on the risk and severity of SARS-CoV-2 infection, association between variations of the genes and susceptibility to diseases has been documented about other infec- 
tious diseases [45]. Further investigations are required to shed light on the importance of gene polymorphisms in innate immunity role-players regarding increased or decreased susceptibility to COVID-19.

Other genes that are involved in the control of inflammatory responses are the components of the Janus kinase (JAK)-signal transducer and activator of transcription (STAT) pathway. The protein families of mammals involved in this pathway include JAK1, JAK2, JAK3 and tyrosine kinase 2 (TYK2) that interact with the cytoplasmic domain of type I and II cytokine receptors and through phosphorylation of the receptors' subunits promulgate cytokine signaling [97,98]. Tyk2 is part of the type I and type III interferon signaling pathways and has critical role in transduction of IFN- $\alpha$ and $-\beta$, IL-6, IL-10, IL-12 and IL-23 signals. IL-12 as an inducer of interferon-gamma (IFN- $\gamma$ ) production, IL-23 as a proinflammatory factor and IL-10 as a critical anti-inflammatory cytokine are involved in the activation of Tyk 2 [99]. Type I IFNs (IFN- $\alpha / \beta)$ are also involved in Tyk 2 activation through binding to their receptor IFNAR. Type I IFNs-Tyk2 interaction elicits the JAK/STAT signaling pathway where phosphorylated STAT1 and STAT2 in complex with IRF lead to the expression of interferon-stimulated genes (ISGs). ISGs encode antiviral proteins that are involved in antiinflammatory response [100]. Considering the importance of Tyk 2 protein in establishing an anti-viral status, the Genetics of Mortality in Critical Care (GenOMICC) and genome-wide association (GWAS) approaches in 2244 patients of intensive care units (ICU) showed that there is a casual link between low expression of IFNAR2 and high expression of TYK2 to severe and lifethreatening COVID-19 [101]. These results represent evidence for a protective role for IFNAR2 in COVID-19 and its loss of function alterations are associated with severe form of the disease [101]. These findings suggest that interferon administration may reduce probability of severe symptoms of the disease. However, administration of exogenous interferon in a large scale clinical trial did not reduce mortality rate in hospitalized patients, proposing that this genetic factor might be mediated in the early steps of disease when SARS-Cov-2 load is high [101].

\section{Host genetic signatures of COVID-19 guide personalized treatment}

To date, no approved effective medication against SARS-CoV-2 infection has been presented, but some potentially repurposable agents such as hydroxychloroquine, melatonin, and chloroquine are investigated by several national and international research groups and some vaccines have been produced [102,103]. However, none of the vaccines provide 100 percent immunity against the virus.

The primary mechanism of preventing COVID-19 infection is to inhibit virus entry through targeting the endosomal pathway. Two known drugs involved in this process are hydroxychloroquine and chloroquine known to increase the $\mathrm{pH}$ of endosomes and inhibit membrane fusion $[103,104]$. Second way of inhibiting viral and host cellular membrane fusion is differential glycosylation of both ACE2 and the S protein. Additionally, several variants of ACE2 which inhibit ACE2 and the S protein interaction may affect the clinical efficiency of chloroquine or hydroxychloroquine, explaining the reason of diverse response of patients to mentioned medicines among different populations $[104,105]$. Another target associated with viral-host cell membrane fusion is TMPRSS2, which is involved in $\mathrm{S}$ protein conformational change. Its blockage by inhibitors such as camostat mesylate in the face of cellular increased $\mathrm{pH}$ environment could be a substantial approach in virus replication inhibition $[59,106]$. It has been proposed that inhibitors of endosomal acidification like CatB/L, E-64D and hydroxychloroquine/chloroquine may have more effect on TMPRSS2-absence patients and might not work or work less for the patients with normal TMPRSS2 enzyme [106]. Considering this, it seems that African and Non-Finnish European populations who carry deleterious variants on TMPRSS2 gene may be more sensitive to the endosomal acidification inhibitors.

Although there are limitations in our understanding of SARS-CoV-2 associated immune signaling pathways, studying and investigating pathways that are involved in excessive inflammatory responses can give us clues on how to overcome the infectious outcomes driven by COVID-19. So, final approach against SARSCoV-2 infection might be to target pathways triggered by the virus. Targeting intracellular molecules instead of viral proteins shows potential advantage as antiviral drugs could be negated by mutations in the virus genome while producing drugs targeting specific signal transducers which is similar in most virus infections can result in effective therapy particularly for the management of COVID-19 [107,108]. Considering structural similarities of SARS-CoV-2 with pathogenic SARS-CoV, it seems that the infection mechanisms of this group of viruses might be identical and may in- 
duce the activation of shared intracellular pathways. It is well stablished that viral RNAs are detected by the pattern recognition receptors such as the family of TLRs (TLR3 and TLR7/8), the cytosolic RNA sensor, and retinoic acid-inducible gene (RIG-I)/MDA5 [109]. Virus recognition result in activation of downstream transduction pathways such as IFN regulatory factor3 (IRF3), nuclear factor $\kappa \mathrm{B}(\mathrm{NF}-\kappa \mathrm{B})$, and JAK/STAT signaling pathways [110].

$\mathrm{NF}-\kappa \mathrm{B}$ as a transcriptional factor is inhibited by the inhibitory proteins $(\mathrm{I} \kappa \mathrm{Bs})$ in the cytoplasm and after activation plays a pivotal role in both innate and adaptive immunity [111]. Pathogens can stimulate $\mathrm{NF}-\kappa \mathrm{B}$ nuclear translocation which subsequently induces the transcription of multiple pro-inflammatory cytokines and chemokines coding genes [112]. In vitro studies demonstrated that SARS-CoV spike protein causes $\mathrm{I} \kappa \mathrm{B} \alpha$ degradation and activates NF- $\kappa \mathrm{B}$ signaling pathway which finally result in induction of IL-6 and TNF $\alpha$ [113]. Interestingly, SARS-CoV-infected mice after treatment with NF- $\kappa$ B inhibitors such as parthenolide, Bay 11-7082 and caffeic acid phenethylester (CAPE) showed inflammation reduction in its lung. Moreover, the survival rate of SARS-CoV-infected mice was increased after pharmacological inhibition of NF$\kappa \mathrm{B}$ [114]. These findings suggest that NF- $\kappa \mathrm{B}$ inhibition may be an effective strategy to counteract pathogenic SARS-CoV-2. In addition, targeting downstream effectors of the pathway like TNF $\alpha$ with anti-TNF $\alpha$ monoclonal antibodies could be another option in dealing with the recent pandemic as TNF $\alpha$ blocking with fliximab and adalimumab result in attenuated inflammatory processes in some inflammatory disorders $[115,116]$. The activity of TNF $\alpha$-converting enzyme (TACE)- dependent shedding of ACE2 is promoted by $\mathrm{S}$ protein of SARS-CoV, so TNF $\alpha$ inhibitors not only attenuate inflammation, but also could prevent virus entry into the cell and inhibit viral infection $[113,117]$.

The JAK/STAT signaling pathway has roles in transducing extracellular signals associated with cytokines, lymphokines and growth factors. Notably, the cytokine IL-6 promotes the downstream activation of JAK/STAT and in turn the activation of this pathway stimulate IL6 expression [118,119]. Abnormal expression of IL-6 promotes the secretion of other cytokines and causes inflammation in the vascular system that is observed in COVID-19 patients [120]. The inflamed vessels produce angiotensin II which induces the synthesis and secretion of IL-6. Activation of JAK/STAT pathway by Angiotensin II receptor type 1 (AT 1 receptor) has been revealed in previous researches and this pattern estab- lishes a positive inflammatory feedback loop [121,122]. The S protein of SARS-CoV downregulates ACE2 expression and causes over-production of angiotensin II. It is speculated that this might also be true in SARS$\mathrm{CoV}-2$ and its $\mathrm{S}$ protein may be involved in overproduction of IL-6 in AT 1/JAK/STAT-dependent manner and subsequently result in vascular inflammation and lung injury $[123,124]$. Additionally, both NF- $\kappa \mathrm{B}$ and ADAM17 are activated by the angiotensin II/AT 1 receptor axis. ADAM17 itself cleave ACE2, as one of its substrate and through ACE2 inactivation, enhances angiotensin II retention [125]. It has been shown that the induction of NF- $\kappa \mathrm{B}$ pathway demands the activation of STAT3, hence SARS-CoV-2 infection could be one of the main activators of STAT3 and NF- $\kappa$ B signaling, which in turn can lead to the IL-6 amplifier mechanism, required for STAT3-mediated hyper-activation of NF- $\kappa \mathrm{B}$ [126]. Considering all these, it is clear that IL-6 is a key driver of cytokine storm and its associated detrimental effects. Therefore, inhibition of either IL-6 itself with monoclonal antibodies like sarilumab and tocilizumab or IL-6/JAK/STAT signaling with baricitinib, fedratinib, and ruxolitinib may attenuate the host inflammatory response associated with SARS-CoV-2 [127].

The sphingosine-1-phosphate (S1P) 1 as second messenger during inflammation play critical role in many immune response processes such as cytokine and chemokine production, lymphocyte trafficking and vascular integrity by binding to five G-protein coupled receptors (S1PRs 1-5) [128]. The S1P 1 receptor which is activated by Ras/ERK, PI3K/Akt/eNOS, and PLC/Ca $2+$ downstream pathways is in association with pathological damage inhibition mediated by the host innate and adaptive immune responses. In animal model affected by influenza virus, S1P 1 agonist blunted cytokine storm particularly TLR3 and TLR7 signaling through inhibiting cytokines and chemokines secretion [108,129]. Moreover, S1P 1 R agonism has also suppressed cytokine and chemokine production by targeting myeloid differentiation primary response gene 88/TIR-domain-containing adapter-inducing IFN$\beta$ (MyD88/TRIF) signaling, two common NF- $\kappa$ B related factors [130]. In this regard, targeting $\mathrm{S} 1 \mathrm{P}$ and $\mathrm{S} 1 \mathrm{P}$ signaling might provide efficient targets to combat SARS-CoV-2 infection.

\section{Vitamin D and COVID-19}

Vitamin D has been found to exert immunomodulatory effects and contribute in induction of anti- 
inflammatory responses, especially in viral infections [131,132]. Moreover, vitamin D can induce expressions of receptors of innate and adaptive immune systems in the respiratory system [133,134]. Low levels of this vitamin in patients with COVID-19 have been shown to be linked with the severe disorder and higher and mortality $[135,136]$. The immunomodulatory and anti-inflammatory effects of optimal serum concentrations of 25-hydroxy vitamin $\mathrm{D}$ have been shown to be beneficial in COVID-19 patients [137]. However, a recent study has shown that administration of high-dose parenteral vitamin D3 did not affect in-hospital mortality rate of critically ill COVID-19 patients with vitamin D deficiency [138].

\section{Role of non-coding RNAs in COVID-19}

Non-coding RNAs, particularly those with regulatory roles have important roles in the pathoetiology of both immune-related and infectious disorders $[85,139,140]$. A number of long non-coding RNAs (lncRNAs) and circular RNAs (circRNAs), particularly those regulating host cell cycle, apoptotic pathways and immune functions have been found to be differentially expressed in COVID-19 patients versus healthy subjects [141]. Moreover, expressions of numerous lncRNAs in SARS-CoV-2-infected cells have been suggested to be controlled by IRF1, IRF4 STAT1 and STAT3. Notably, these lncRNAs may participate in antiviral responses [142].

\section{Conclusion}

COVID-19 pandemic has affected different populations with diverse pattern, as the number of total affected cases per one million people in some countries with developed health care system is more than other geographic locations with under developed health care systems. Moreover, susceptibility of people to the virus within populations and even among members of a family is different as some patients show mild symptoms of the diseases, while others experience moderate symptoms and some cases are critically ill. These evidences suggest that several factors such as multiple virus variants and host genetics might play critical role in making people more prone/resistant to the infection. In this study we tried to cast light on the SARS-CoV-2 replication cycle, its driven signaling pathways and their associated proteins with the aim of understanding mecha- nisms involved in the disease pathogenesis. We propose that targeting specific proteins associated with virus entrance and replication as well as host immune response can accelerate development of therapeutic agents and subsequently reduce mortality rate among different populations and ethnic groups. However, intense and profound studies are needed to investigate each angle of proposed approaches in managing the pandemic and confirm such a conclusion.

As an active area of investigation, variants in SARSCoV-2 and its associated host genes should be classified in different categories such as asymptomatic, moderately symptomatic and severely symptomatic with urgent hospitalization. Providing such a classification and identifying genomics and genetic pathways associated with SARS-CoV-2 susceptibility may help clinicians in managing further waves of the pandemic. At the end, we encourage all scientists to assess gene expression and also variants among individuals with severe COVID-19 using novel massive parallel sequencing methods and other new approaches.

\section{Future perspectives}

Future high throughput sequencing strategies as well as genome-wide association studies in SARS-CoV-2infected individuals and assessment of association between putative genetic variants/differentially expressed genes and disease course would facilitate identification of the role of genetic factors in this regard.

\section{References}

[1] N. Zhu et al., A novel coronavirus from patients with pneumonia in China, 2019. New England Journal of Medicine 2020.

[2] R.L. Kruse, Therapeutic strategies in an outbreak scenario to treat the novel coronavirus originating in Wuhan, China, F1000Research 9 (2020).

[3] H.M. Ashour, W.F. Elkhatib, M. Rahman and H.A. Elshabrawy, Insights into the recent 2019 novel coronavirus (SARS-CoV-2) in light of past human coronavirus outbreaks, Pathogens 9 (2020), 186.

[4] P.S. Masters, The molecular biology of coronaviruses, $\mathrm{Ad}$ vances in Virus Research 66 (2006), 193-292.

[5] J. Sui et al., Potent neutralization of severe acute respiratory syndrome (SARS) coronavirus by a human $\mathrm{mAb}$ to $\mathrm{S} 1$ protein that blocks receptor association, Proceedings of the National Academy of Sciences 101 (2004), 2536-2541.

[6] C. Sohrabi et al., World Health Organization declares global emergency: A review of the 2019 novel coronavirus (COVID19), International Journal of Surgery, 2020. 
[7] P.C. Woo et al., Discovery of seven novel Mammalian and avian coronaviruses in the genus deltacoronavirus supports bat coronaviruses as the gene source of alphacoronavirus and betacoronavirus and avian coronaviruses as the gene source of gammacoronavirus and deltacoronavirus, Journal of Virology 86 (2012), 3995-4008

[8] A. Mittal et al., COVID-19 pandemic: Insights into structure, function, and hACE2 receptor recognition by SARS-CoV-2, PLoS Pathogens 16 (2020), e1008762.

[9] A. Wu et al., Genome composition and divergence of the novel coronavirus (2019-nCoV) originating in China, Cell host \& microbe, 2020.

[10] B.H. Harcourt et al., Identification of severe acute respiratory syndrome coronavirus replicase products and characterization of papain-like protease activity, Journal of Virology 78 (2004), 13600-13612.

[11] J.F.-W. Chan et al., Genomic characterization of the 2019 novel human-pathogenic coronavirus isolated from a patient with atypical pneumonia after visiting Wuhan, Emerging Microbes \& Infections 9 (2020), 221-236.

[12] E. Teixeira Prates et al., Potential pathogenicity determinants identified from structural proteomics of SARS-CoV and SARS-CoV-2, (Oak Ridge National Lab. (ORNL), Oak Ridge, TN (United States), 2020).

[13] B.J. Bosch, R. Van der Zee, C.A. De Haan and P.J. Rottier, The coronavirus spike protein is a class I virus fusion protein: Structural and functional characterization of the fusion core complex, Journal of Virology 77 (2003), 8801-8811.

[14] A.C. Walls et al., Structure, function, and antigenicity of the SARS-CoV-2 spike glycoprotein, Cell, 2020.

[15] Q. Wang et al., Structural and functional basis of SARS-CoV2 entry by using human ACE2, Cell, 2020.

[16] B. Nal et al., Differential maturation and subcellular localization of severe acute respiratory syndrome coronavirus surface proteins S, M and E, Journal of General Virology 86 (2005), 1423-1434.

[17] D. Schoeman and B.C. Fielding, Coronavirus envelope protein: Current knowledge, Virology Journal 16 (2019), 69.

[18] Y. Siu et al., The M, E, and N structural proteins of the severe acute respiratory syndrome coronavirus are required for efficient assembly, trafficking, and release of virus-like particles, Journal of Virology 82 (2008), 11318-11330.

[19] J.L. Nieto-Torres et al., Subcellular location and topology of severe acute respiratory syndrome coronavirus envelope protein, Virology 415 (2011), 69-82.

[20] S. Mukherjee, D. Bhattacharyya and A. Bhunia, Hostmembrane interacting interface of the SARS coronavirus envelope protein: Immense functional potential of C-terminal domain, Biophysical Chemistry 266 (2020), 106452.

[21] C.-k. Chang et al., Modular organization of SARS coronavirus nucleocapsid protein, Journal of Biomedical Science 13 (2006), 59-72.

[22] K.R. Hurst, C.A. Koetzner and P.S. Masters, Identification of in vivo-interacting domains of the murine coronavirus nucleocapsid protein, Journal of Virology 83 (2009), 72217234.

[23] C. Wang et al., The establishment of reference sequence for SARS-CoV-2 and variation analysis, Journal of Medical Virology 92 (2020), 667-674.

[24] D. Wrapp et al., Cryo-EM structure of the 2019-nCoV spike in the prefusion conformation, Science 367 (2020), 12601263.

[25] D. Wang et al., Immunoinformatic analysis of T-and B-cell epitopes for SARS-CoV-2 vaccine design, Vaccines 8 (2020),
355.

[26] J. Lan et al., Structure of the SARS-CoV-2 spike receptorbinding domain bound to the ACE2 receptor, Nature 581 (2020), 215-220.

27] R. Yan et al., Structural basis for the recognition of SARSCoV-2 by full-length human ACE2, Science 367 (2020), 1444-1448.

[28] S.K. Wong, W. Li, M.J. Moore, H. Choe and M. Farzan, A 193-amino acid fragment of the SARS coronavirus S protein efficiently binds angiotensin-converting enzyme 2. Journal of Biological Chemistry 279 (2004), 3197-3201.

[29] Y. He et al., Identification and characterization of novel neutralizing epitopes in the receptor-binding domain of SARSCoV spike protein: Revealing the critical antigenic determinants in inactivated SARS-CoV vaccine, Vaccine 24 (2006), 5498-5508.

[30] P. Hidalgo, M. Valdés and R.A. González, Molecular biology of coronaviruses: An overview of virus-host interactions and pathogenesis, Boletin Medico del Hospital Infantil de Mexico 78 (2021), 41-58.

[31] S. Matsuyama et al., Efficient activation of the severe acute respiratory syndrome coronavirus spike protein by the transmembrane protease TMPRSS2, Journal of Virology 84 (2010), 12658-12664.

[32] J.K. Millet and G.R. Whittaker, Host cell proteases: Critical determinants of coronavirus tropism and pathogenesis, Virus Research 202 (2015), 120-134.

[33] B. Coutard et al., The spike glycoprotein of the new coronavirus 2019-nCoV contains a furin-like cleavage site absent in CoV of the same clade, Antiviral Research 176 (2020), 104742 .

[34] B.J. Bosch, W. Bartelink and P.J. Rottier, Cathepsin L functionally cleaves the severe acute respiratory syndrome coronavirus class I fusion protein upstream of rather than adjacent to the fusion peptide, Journal of Virology 82 (2008), 8887-8890.

[35] H.A. Elshabrawy et al., Identification of a broad-spectrum antiviral small molecule against severe acute respiratory syndrome coronavirus and Ebola, Hendra, and Nipah viruses by using a novel high-throughput screening assay, Journal of Virology 88 (2014), 4353-4365.

[36] G. Simmons et al., Characterization of severe acute respiratory syndrome-associated coronavirus (SARS-CoV) spike glycoprotein-mediated viral entry, Proceedings of the $\mathrm{Na}$ tional Academy of Sciences 101 (2004), 4240-4245.

[37] P. V'kovski, A. Kratzel, S. Steiner, H. Stalder and V. Thiel, Coronavirus biology and replication: Implications for SARS CoV-2, Nature Reviews Microbiology 19 (2021), 155-170.

[38] S. Kumar, R. Nyodu, V.K. Maurya and S.K. Saxena, in Coronavirus Disease 2019 (COVID-19), Springer, 2020, 23-31.

[39] L.a. Alanagreh, F. Alzoughool and M. Atoum, The human coronavirus disease COVID-19: Its origin, characteristics, and insights into potential drugs and its mechanisms, Pathogens 9 (2020), 331

[40] R. Mirzaei et al., A contemporary review on pathogenesis and immunity of COVID-19 infection, Molecular Biology Reports 47 (2020), 5365-5376.

[41] L. Mousavizadeh and S. Ghasemi, Genotype and phenotype of COVID-19: Their roles in pathogenesis, Journal of Microbiology, Immunology and Infection, 2020.

[42] S. Ghafouri-Fard et al., Effects of host genetic variations on response to, susceptibility and severity of respiratory infections, Biomedicine \& Pharmacotherapy $=$ Biomedecine $\&$ Pharmacotherapie 128 (2020), 110296. 
[43] S. Ghafouri-Fard et al., Angiotensin converting enzyme: A review on expression profile and its association with human disorders with special focus on SARS-CoV-2 infection, Vascular Pharmacology 130 (2020), 106680.

[44] A.R. Bourgonje et al., Angiotensin-converting enzyme-2 (ACE2), SARS-CoV-2 and pathophysiology of coronavirus disease 2019 (COVID-19), The Journal of Pathology, 2020.

[45] M. Debnath, M. Banerjee and M. Berk, Genetic gateways to COVID-19 infection: Implications for risk, severity, and outcomes, The FASEB Journal, 2020.

[46] Y. Hou et al., New insights into genetic susceptibility of COVID-19: An ACE2 and TMPRSS2 polymorphism analysis, BMC Medicine 18 (2020), 1-8.

[47] W. Li et al., Receptor and viral determinants of SARScoronavirus adaptation to human ACE2, The EMBO Journal 24 (2005), 1634-1643.

[48] A. Heurich et al., TMPRSS2 and ADAM17 cleave ACE2 differentially and only proteolysis by TMPRSS2 augments entry driven by the severe acute respiratory syndrome coronavirus spike protein, Journal of Virology 88 (2014), 1293-1307.

[49] R. Reddy Gaddam, S. Chambers and M. Bhatia, ACE and ACE2 in inflammation: A tale of two enzymes, Inflammation \& Allergy-Drug Targets (Formerly Current Drug TargetsInflammation \& Allergy) 13 (2014), 224-234.

[50] M.C. Thomas et al., Genetic Ace2 deficiency accentuates vascular inflammation and atherosclerosis in the ApoE knockout mouse, Circulation Research 107 (2010), 888-897.

[51] M.-Y. Li, L. Li, Y. Zhang and X.-S. Wang, Expression of the SARS-CoV-2 cell receptor gene ACE2 in a wide variety of human tissues, Infectious Diseases of Poverty 9 (2020), 1-7.

[52] F. Zhou et al., Clinical course and risk factors for mortality of adult inpatients with COVID-19 in Wuhan, China: a retrospective cohort study, The lancet, 2020.

[53] Y. Chen and L. Li, SARS-CoV-2: Virus dynamics and host response, The Lancet Infectious Diseases 20 (2020), 515516

[54] R. Verity et al., Estimates of the severity of coronavirus disease 2019: a model-based analysis, The Lancet Infectious Diseases, 2020.

[55] H. Cai, Sex difference and smoking predisposition in patients with COVID-19, The Lancet Respiratory Medicine 8 (2020), e20.

[56] S.L. Klein and K.L. Flanagan, Sex differences in immune responses, Nature Reviews Immunology 16 (2016), 626.

[57] M.C. White, R. Fleeman and A.C. Arnold, Sex differences in the metabolic effects of the renin-angiotensin system, Biology of Sex Differences 10 (2019), 31.

[58] J.D. Strope and C.H. Chau, TMPRSS2: Potential Biomarker for COVID-19 Outcomes, Journal of Clinical Pharmacology, 2020.

[59] M. Hoffmann et al., SARS-CoV-2 cell entry depends on ACE2 and TMPRSS2 and is blocked by a clinically proven protease inhibitor, Cell, 2020.

[60] I. Gupta, B. Rizeq, E. Elkord, S. Vranic and A.-E. A Moustafa, SARS-CoV-2 infection and lung cancer: Potential therapeutic modalities, Cancers 12 (2020), 2186.

[61] S. Bertram et al., Influenza and SARS-coronavirus activating proteases TMPRSS2 and HAT are expressed at multiple sites in human respiratory and gastrointestinal tracts, PloS One 7 (2012), e35876.

[62] J. Lucas et al., The androgen-regulated type II serine protease TMPRSS2 is differentially expressed and mislocalized in prostate adenocarcinoma, The Journal of Pathology: A Jour- nal of the Pathological Society of Great Britain and Ireland 215 (2008), 118-125.

[63] M. Madjid, P. Safavi-Naeini, S.D. Solomon and O. Vardeny, Potential effects of coronaviruses on the cardiovascular system: a review, JAMA cardiology, 2020.

[64] X.-w. Pan, H.Z. Da Xu, W. Zhou, L.-h. Wang and X.-g. Cui, Identification of a potential mechanism of acute kidney injury during the COVID-19 outbreak: a study based on single-cell transcriptome analysis, Intensive care medicine, 2020, 1.

[65] B.A. Schuler et al., Age-determined expression of priming protease TMPRSS2 and localization of SARS-CoV-2 infection in the lung epithelium, bioRxiv, 2020.

[66] H. Mostafavi et al., Identifying genetic variants that affect viability in large cohorts, PLoS Biology 15 (2017), e2002458.

[67] Y. Xing, X. Li, X. Gao and Q. Dong, Natural Polymorphisms Are Present in the Furin Cleavage Site of the SARS-CoV-2 Spike Glycoprotein, Frontiers in Genetics 11 (2020), 783.

[68] M. Hoffmann, H. Kleine-Weber and S. Pöhlmann, A multibasic cleavage site in the spike protein of SARS-CoV-2 is essential for infection of human lung cells, Molecular Cell, 2020.

[69] M.S. Sarac, J.R. Peinado, S.H. Leppla and I. Lindberg, Protection against anthrax toxemia by hexa-D-arginine in vitro and in vivo, Infection and Immunity 72 (2004), 602-605.

[70] L. Torre-Fuentes et al., ACE2, TMPRSS2, and Furin variants and SARS-CoV-2 infection in Madrid, Spain, Journal of medical virology, 2020

[71] J.M. Blackwell, S.E. Jamieson and D. Burgner, HLA and infectious diseases, Clinical Microbiology Reviews 22 (2009), 370-385.

[72] A. Sanchez-Mazas, HLA studies in the context of coronavirus outbreaks, Swiss Medical Weekly 150 (2020).

[73] E.J. Giamarellos-Bourboulis et al., Complex immune dysregulation in COVID-19 patients with severe respiratory failure, Cell host \& microbe, 2020.

[74] A. Nguyen et al., Human leukocyte antigen susceptibility map for SARS-CoV-2, Journal of virology, 2020.

[75] J. Romero-López et al., Prediction of SARS-CoV2 spike protein epitopes reveals HLA-associated susceptibility, 2020.

[76] K.M. Campbell, G. Steiner, D.K. Wells, A. Ribas and A. Kalbasi, Prediction of SARS-CoV-2 epitopes across 9360 HLA class I alleles, BioRxiv, 2020.

[77] S.F. Ahmed, A.A. Quadeer and M.R. McKay, Preliminary identification of potential vaccine targets for the COVID-19 coronavirus (SARS-CoV-2) based on SARS-CoV immunological studies, Viruses 12 (2020), 254

[78] A. Saxena et al., HLA-A* 02 repertoires in three defined population groups from North and Central India: Punjabi Khatries, Kashmiri Brahmins and Sahariya Tribe, Hla 93 (2019), 16-23.

[79] K. Cao et al., Differentiation between African populations is evidenced by the diversity of alleles and haplotypes of HLA class I loci, Tissue Antigens 63 (2004), 293-325.

[80] F.F. González-Galarza et al., Allele frequency net 2015 update: New features for HLA epitopes, KIR and disease and HLA adverse drug reaction associations, Nucleic Acids Research 43 (2015), D784-D788.

[81] S.H. Nile et al., COVID-19: Pathogenesis, cytokine storm and therapeutic potential of interferons, Cytokine \& Growth Factor Reviews, 2020.

[82] P. Mehta et al., COVID-19: Consider cytokine storm syndromes and immunosuppression, Lancet (London, England) 395 (2020), 1033. 
[83] C. Huang et al., Clinical features of patients infected with 2019 novel coronavirus in Wuhan, China, The lancet 395 (2020), 497-506.

[84] H.A. Rothan and S.N. Byrareddy, The epidemiology and pathogenesis of coronavirus disease (COVID-19) outbreak, Journal of autoimmunity, 2020, 102433.

[85] M. Taheri et al., Evaluation of expression of VDR-associated lncRNAs in COVID-19 patients, BMC Infectious Diseases 21 (2021), 588.

[86] S.C. Hoffmann et al., Ethnicity greatly influences cytokine gene polymorphism distribution, American Journal of Transplantation 2 (2002), 560-567.

[87] K. Ozato, H. Tsujimura and T. Tamura, Toll-like receptor signaling and regulation of cytokine gene expression in the immune system, Biotechniques 33 (2002), S66-S75.

[88] P.K. Manchanda, H.K. Bid and R.D. Mittal, Ethnicity greatly influences the interleukin-1 gene cluster (IL-1b promoter, exon-5 and IL-1Ra) polymorphisms: a pilot study of a north Indian population, 2005.

[89] R. Noroozi et al., Altered cytokine levels and immune responses in patients with SARS-CoV-2 infection and related conditions, Cytokine 133 (2020), 155143

[90] W.P. Chong et al., The interferon gamma gene polymorphism $+874 \mathrm{~A} / \mathrm{T}$ is associated with severe acute respiratory syndrome, BMC Infectious Diseases 6 (2006), 1-4

[91] F. Tang et al., IL-12 RB1 genetic variants contribute to human susceptibility to severe acute respiratory syndrome infection among Chinese, PloS One 3 (2008), e2183.

[92] A.L. Totura et al., Toll-like receptor 3 signaling via TRIF contributes to a protective innate immune response to severe acute respiratory syndrome coronavirus infection, MBio 6 (2015).

[93] L.E. Gralinski et al., Allelic variation in the toll-like receptor adaptor protein ticam 2 contributes to SARS-coronavirus pathogenesis in mice, G3: Genes, Genomes, Genetics 7 (2017), 1653-1663.

[94] L.B. Barreiro et al., Evolutionary dynamics of human Tolllike receptors and their different contributions to host defense, PLoS Genet 5 (2009), e1000562.

[95] T. Gao et al., Highly pathogenic coronavirus N protein aggravates lung injury by MASP-2-mediated complement overactivation, MedRxiv, 2020

[96] A.H. de Nooijer et al., Complement Activation in the Disease Course of Coronavirus Disease 2019 and Its Effects on Clinical Outcomes, The Journal of Infectious Diseases, 2020

[97] C. Vogl et al., Transcriptome analysis reveals a major impact of JAK protein tyrosine kinase 2 (Tyk2) on the expression of interferon-responsive and metabolic genes, BMC Genomics 11 (2010), 199.

[98] X. Min et al., Structural and functional characterization of the JH2 pseudokinase domain of JAK family tyrosine kinase 2 (TYK2), Journal of Biological Chemistry 290 (2015), 2726127270.

[99] M.H. Shaw et al., Tyk2 negatively regulates adaptive Th1 immunity by mediating IL-10 signaling and promoting IFN$\gamma$-dependent IL-10 reactivation, The Journal of Immunology 176 (2006), 7263-7271.

[100] C. Xia, P. Anderson and B. Hahm, Viral dedication to vigorous destruction of interferon receptors, Virology 522 (2018), 19-26.

[101] E. Pairo-Castineira et al., Genetic mechanisms of critical illness in Covid-19, Nature, 2020, 1-1.

[102] Y. Zhou et al., Network-based drug repurposing for novel coronavirus 2019-nCoV/SARS-CoV-2, Cell Discovery 6
(2020), 1-18.

[103] J.M. Sanders, M.L. Monogue, T.Z. Jodlowski and J.B. Cutrell, Pharmacologic treatments for coronavirus disease 2019 (COVID-19): A review, Jama 323 (2020), 1824-1836.

[104] A. Savarino, L. Di Trani, I. Donatelli, R. Cauda and A. Cassone, New insights into the antiviral effects of chloroquine, The Lancet Infectious Diseases 6 (2006), 67-69.

[105] E.S. Rosenberg et al., Association of treatment with hydroxychloroquine or azithromycin with in-hospital mortality in patients with COVID-19 in New York state, Jama, 2020.

[106] A. Shulla et al., A transmembrane serine protease is linked to the severe acute respiratory syndrome coronavirus receptor and activates virus entry, Journal of Virology 85 (2011), 873882.

[107] M. Catanzaro et al., Immune response in COVID-19: Addressing a pharmacological challenge by targeting pathways triggered by SARS-CoV-2, Signal Transduction and Targeted Therapy 5 (2020), 1-10.

[108] J.R. Teijaro et al., Endothelial cells are central orchestrators of cytokine amplification during influenza virus infection, Cell 146 (2011), 980-991.

[109] M. de Marcken, K. Dhaliwal, A.C. Danielsen, A.S. Gautron and M. Dominguez-Villar, TLR7 and TLR8 activate distinct pathways in monocytes during RNA virus infection, Science Signaling 12 (2019)

[110] J. Olejnik, A.J. Hume and E. Mühlberger, Toll-like receptor 4 in acute viral infection: Too much of a good thing, PLoS Pathogens 14 (2018), e1007390.

[111] Q. Li and I.M. Verma, NF- $\kappa \mathrm{B}$ regulation in the immune system, Nature Reviews Immunology 2 (2002), 725-734.

[112] M. Hayden, A. West and S. Ghosh, NF- $\kappa$ B and the immune response, Oncogene 25 (2006), 6758-6780.

[113] W. Wang et al., Up-regulation of IL- 6 and TNF- $\alpha$ induced by SARS-coronavirus spike protein in murine macrophages via NF- $\kappa$ B pathway, Virus Research 128 (2007), 1-8.

[114] M.L. DeDiego et al., Inhibition of NF- $\kappa$ B-mediated inflammation in severe acute respiratory syndrome coronavirusinfected mice increases survival, Journal of Virology $\mathbf{8 8}$ (2014), 913-924.

[115] L.C. Silva, L.C. Ortigosa and G. Benard, Anti-TNF- $\alpha$ agents in the treatment of immune-mediated inflammatory diseases: Mechanisms of action and pitfalls, Immunotherapy 2 (2010), 817-833.

[116] G. Lapadula et al., Adalimumab in the treatment of immunemediated diseases, International Journal of Immunopathology and Pharmacology 27 (2014), 33-48.

[117] S. Haga et al., Modulation of TNF- $\alpha$-converting enzyme by the spike protein of SARS-CoV and ACE2 induces TNF$\alpha$ production and facilitates viral entry, Proceedings of the National Academy of Sciences 105 (2008), 7809-7814.

[118] C. Zhang, Z. Wu, J.-W. Li, H. Zhao and G.-Q. Wang, The cytokine release syndrome (CRS) of severe COVID-19 and Interleukin-6 receptor (IL-6R) antagonist Tocilizumab may be the key to reduce the mortality, International journal of antimicrobial agents, 2020, 105954 .

[119] C. Lee et al., Janus kinase-signal transducer and activator of transcription mediates phosphatidic acid-induced interleukin (IL) $-1 \beta$ and IL-6 production, Molecular Pharmacology 69 (2006), 1041-1047.

[120] D. Qu, J. Liu, C.W. Lau and Y. Huang, IL-6 in diabetes and cardiovascular complications, British Journal of Pharmacology 171 (2014), 3595-3603.

[121] B. Schieffer et al., Role of NAD (P) H oxidase in angiotensin II - induced JAK/STAT signaling and cytokine induction, 
Circulation Research 87 (2000), 1195-1201.

[122] M.B. Marrero et al., Direct stimulation of Jak/STAT pathway by the angiotensin II AT 1 receptor, Nature 375 (1995), 247250

[123] K. Kuba et al., A crucial role of angiotensin converting enzyme 2 (ACE2) in SARS coronavirus-induced lung injury, Nature Medicine 11 (2005), 875-879.

[124] I. Glowacka et al., Differential downregulation of ACE2 by the spike proteins of severe acute respiratory syndrome coronavirus and human coronavirus NL63, Journal of Virology 84 (2010), 1198-1205

[125] S. Eguchi, T. Kawai, R. Scalia and V. Rizzo, Understanding angiotensin II type 1 receptor signaling in vascular pathophysiology, Hypertension 71 (2018), 804-810.

[126] M. Murakami, D. Kamimura and T. Hirano, Pleiotropy and specificity: Insights from the interleukin 6 family of cytokines, Immunity 50 (2019), 812-831.

[127] G.A.M. Sanchez et al., JAK $1 / 2$ inhibition with baricitinib in the treatment of autoinflammatory interferonopathies, The Journal of Clinical Investigation 128 (2018), 3041-3052.

[128] S. Spiegel and S. Milstien, The outs and the ins of sphingosine-1-phosphate in immunity, Nature Reviews Immunology 11 (2011), 403-415.

[129] A.M. Bryan and M. Del Poeta, Sphingosine-1-phosphate receptors and innate immunity, Cellular Microbiology 20 (2018), e12836.

[130] J.R. Teijaro, K.B. Walsh, S. Rice, H. Rosen and M.B. Oldstone, Mapping the innate signaling cascade essential for cytokine storm during influenza virus infection, Proceedings of the National Academy of Sciences 111 (2014), 3799-3804.

[131] N. Charoenngam and M.F. Holick, Immunologic effects of vitamin D on human health and disease, Nutrients 12 (2020), 2097.

[132] W.B. Grant et al., Evidence that vitamin D supplementation could reduce risk of influenza and COVID-19 infections and deaths, Nutrients 12 (2020), 988.
[133] C.L. Greiller et al., Vitamin D attenuates rhinovirus-induced expression of intercellular adhesion molecule-1 (ICAM-1) and platelet-activating factor receptor (PAFR) in respiratory epithelial cells, The Journal of Steroid Biochemistry and Molecular Biology 187 (2019), 152-159.

[134] R.A. Brockman-Schneider, R.J. Pickles and J.E. Gern, Effects of vitamin D on airway epithelial cell morphology and rhinovirus replication, PLoS One 9 (2014), e86755.

[135] R. Munshi et al., Vitamin D insufficiency as a potential culprit in critical COVID-19 patients, Journal of Medical Virology 93 (2021), 733-740.

[136] E. Merzon et al., Low plasma $25(\mathrm{OH})$ vitamin D level is associated with increased risk of COVID-19 infection: an Israeli population-based study, The FEBS Journal 287 (2020), 3693-3702.

[137] F. Mitchell, Vitamin-D and COVID-19: Do deficient risk a poorer outcome? The Lancet. Diabetes \& Endocrinology 8 (2020), 570.

[138] M. Güven and H. Gültekin, The effect of high-dose parenteral vitamin $\mathrm{D}(3)$ on COVID-19-related inhospital mortality in critical COVID-19 patients during intensive care unit admission: An observational cohort study, European Journal of Clinical Nutrition 75 (2021), 1383-1388.

[139] S. Shirahama, A. Miki, T. Kaburaki and N. Akimitsu, Long non-coding RNAs involved in pathogenic infection, Frontiers in Genetics 11 (2020), 454.

[140] J. Chen, L. Ao and J. Yang, Long non-coding RNAs in diseases related to inflammation and immunity, Annals of Translational Medicine 7 (2019)

[141] Y. Wu et al., A study of differential circRNA and lncRNA expressions in COVID-19-infected peripheral blood, Scientific Reports 11 (2021), 1-14.

[142] S. Laha et al., In silico analysis of altered expression of long non-coding RNA in SARS-CoV-2 infected cells and their possible regulation by STAT1, STAT3 and interferon regulatory factors, Heliyon 7 (2021), e06395. 